\title{
Position of myopic iris-claw phakic intraocular lens by Scheimpflug photography and ultrasound biomicroscopy
}

A-Y Yu, Z-D Lin, X-Q Chen, X-Y Cai, Y-Z Liu and S-K Luo

\begin{abstract}
Purpose To evaluate the position of iris-claw phakic intraocular lens (ICPIOL) in highly myopic eyes by Scheimpflug photography (SP) and ultrasound biomicroscopy (UBM). Methods Nine eyes of five patients aged $31 \pm 10$ years with average spherical equivalent of $-16.04 \pm 5.46 \mathrm{D}$ (range -7.88 to $-22.88 \mathrm{D}$ ) were enrolled in this prospective study and implanted with Verisyse ICPIOLs (AMO). The anterior segment was evaluated by SP and UBM preoperatively and for at least 1 month postoperatively. The statistical significance may be questionable due to the limited number (nine) of eyes.

Results By SP and UBM, the distance
\end{abstract} between corneal endothelium and lens (anterior chamber depth) preoperatively was $3.10 \pm 0.14$ and $3.07 \pm 0.11 \mathrm{~mm}$, respectively; between ICPIOL and corneal endothelium (pseudo-anterior chamber depth), $1.88 \pm 0.09$ and $1.99 \pm 0.12 \mathrm{~mm}$, respectively; between lens and posterior surface of ICPIOL (IL), $0.76 \pm 0.13$ and $0.67 \pm 0.06 \mathrm{~mm}$, respectively; between superior optic edge and iris (SOEI), $0.23 \pm 0.23$ and $0.58 \pm 0.24 \mathrm{~mm}$, respectively; between inferior optic edge and iris (IOEI), $0.07 \pm 0.13$ and $0.41 \pm 0.22 \mathrm{~mm}$, respectively; between ICPIOL haptics and the angle of anterior chamber (HA), $0.90 \pm 0.17$ and $1.45 \pm 0.13 \mathrm{~mm}$, respectively. ACD was well correlated between the two methods, but PACD, IL, OEI, HA were not. The postoperative measures, except IL, were significantly different between the two methods.

Conclusion The differences between measurements by SP and UBM reveal the
ICPIOL's position variations with change of body position. Nevertheless, it seems adequate that space is maintained between ICPIOL and corneal endothelium, angle, and crystalline lens. The ICPIOL implanted in phakic eyes seems a safe alternative for treatment of high myopia.

Eye (2008) 22, 233-239; doi:10.1038/sj.eye.6702829; published online 13 April 2007

Keywords: myopia; refractive surgery; phakic intraocular lens; Scheimpflug photography; ultrasound biomicroscopy; safety

\section{Introduction}

The optical superiority of iris-claw phakic intraocular lens (ICPIOL) as well as the obtained correction and refractive stability compared with other forms of surgery for high myopia have been recently reported in several studies. ${ }^{1-6}$ Because of the close proximity of the ICPIOL and intraocular structures, some authors, ${ }^{7-9}$ however, have expressed concern about the potential for the ICPIOL to endothelial cell reduction resulting from contact between the ICPIOL and cornea, iritis due to contact with the delicate iris tissue, and secondary glaucoma due to pupillary block or pigment dispersion. Precise evaluation of ICPIOL's position in the patient with high myopia in different body positions plays an important role in early detection of ICPIOL-intraocular tissue interaction and prevention in potential complications. Although slit lamp is a useful instrument by which clinicians evaluate the position of ICPIOL, it
Zhongshan Ophthalmic Center, Sun Yat-Sen University, Guangzhou, China

Correspondence: A-Y Yu, Zhongshan Ophthalmic

Center,

Sun Yat-sen

University, 54 Xianlie Nan Road,

Guangzhou, 510060, PR China

Tel: + 862087330294 ;

Fax: + 8613868760001

E-mail: yaybetter@

yahoo.com.cn

Received: 19 January 2006 Accepted in revised form: 16 March 2007 Published online: 13 April 2007 
does not allow precise quantitive observations, and cannot be performed in supine position. Scheimpflug photography (SP) and ultrasound biomicroscopy (UBM), capable of measuring the anterior segment with high reliability use different techniques, and each has its own advantages and disadvantages. To our knowledge, although SP and UBM have been assessed separately in a number of studies with different study populations, no study of both instruments in same patients implanted with ICPIOLs has been published. The present study investigated the position of the myopic ICPIOL relative to adjacent structures in the anterior segment in upright and supine position using SP and UBM. The measurements by the two methods were compared, and the ICPIOL's position variations with change of body position were evaluated in vivo.

\section{Patients and methods}

\section{Patients}

The Verisyse ICPIOL (AMO) was implanted in nine eyes of five Chinese patients with age of $31 \pm 10$ years (range 19-46 years) from May to November 2005, including three women (six eyes) and two men (three eyes), four right eyes and five left eyes. All patients revealed no abnormal findings by a comprehensive ophthalmic screening examination including gonioscopy and iris examination performed before surgery, and $67 \pm 30$ days (range 31-115 days) since implantation of the ICPIOL. In all nine eyes, the anterior segment parameters were first measured with SP, followed by UBM, both without mydriasis.

The research protocol followed the tenets of the Helsinki Declaration and was approved by the Local Ethics Committee. All patients were fully informed about the details and possible risks inherent to the surgery and to this study. Written informed consents were obtained from all patients.

\section{ICPIOL implantation}

ICPIOL power was calculated according to the manufacturer's instructions. However, the VRSM60 lens model (optic diameter of $6.0 \mathrm{~mm}$ ) is available up to $-15.0 \mathrm{D}$. If higher ICPIOL power more than $-15.0 \mathrm{D}$ was required, the VRSM50 lens model (optic diameter of $5.0 \mathrm{~mm}$ ) was used. The ICPIOL implantation was performed following a standard protocol. Corneoscleral incisions of 5.5 or $6.0 \mathrm{~mm}$ were made at 12 o'clock and two paracenteses were placed at 9 and 3 o'clock. The ICPIOL was inserted with the pupil constricted and the anterior chamber filled with viscoelastic material. After it was introduced into the anterior chamber, the ICPIOL was rotated with the haptics at 3 and 9 o'clock. The optic was centred over the pupil. Iris entrapment was performed by holding a knuckle of iris with a specially designed implantation forceps (P1318B, Duckworth \& Kent) while gently pressing the centre of one haptic over the knuckle, thus grasping iris tissue with both claws. The same procedure was repeated with the other haptic. Once the ICPIOL is fixated, a single peripheral iridectomy was performed manually at 1 or 11 o'clock. The corneoscleral wound was closed with sutures (10-0 Nylon), and the viscoelastic agent was removed by manual irrigation. All ICPIOLs were implanted by the same surgeon (Z.L.).

\section{SP examination}

Preoperative and postoperative examinations were performed using the Pentacam Scheimpflug anterior eye segment analysis system (Oculus, Germany). The headrest and chinrest of the system ensured that the patient's head was in the same upright position for each follow-up examination. For the examination, the patient was asked to fixate on a target inside the device, and the reflection of the fixation light was centred in the pupil automatically to ensure that the same section was obtained. Fifty cross-sectional images were taken through the cornea, iris, ICPIOL, and crystalline lens, providing $360^{\circ}$ coverage of the anterior chamber, as described by the manufacturer.

In all the eyes, after the distortion of images was corrected as described by Coppens et al,$^{10}$ the following parameters were measured: the preoperative distance between the corneal endothelium and the anterior surface of the lens capsule (ACD), which was measured two times to assess the reliability of the method; the distance between the corneal endothelium and the anterior surface of the ICPIOL (pseudo-ACD, PACD); the distance between the posterior surface of the ICPIOL and the anterior surface of the lens capsule (IL); the distance between the superior/inferior optic edge and the iris (S/IOEI); the shortest distance between the farthest point of the ICPIOL haptics and the angle of anterior chamber (HA). All measurements were made using the calipers provided by the Pentacam Scheimpflug system software on the two-dimensional image. All eyes were scanned by a single operator.

\section{UBM examination}

Preoperative and postoperative examinations were performed using the Humphrey ultrasound biomicroscope, model 840 . This system uses a $50 \mathrm{MHz}$ transducer with a probe and has an axial and lateral resolution of $50 \mu \mathrm{m}$ that provides a sampling resolution 
of $5 \mu \mathrm{m}$ on scans, as described by the manufacturer. For the examination, the patient was maintained in a supine position with instillation of topical proparacaine $0.5 \%$ (Alcaine). Depending on the size of the eye's aperture, a 20 or $22 \mathrm{~mm}$ eyecup filled with a sterile gonioscopic solution was inserted between the upper and lower eyelids. The patient was asked to fixate on a ceiling target with the fellow eye to maintain accommodation and fixation. Cross-sectional images were taken through the cornea, iris, ciliary body, and lens, starting from the nasal to the temporal side and from the superior to the inferior side, providing $360^{\circ}$ coverage of the anterior chamber. It should be emphasized that, given the high chance of eye movements (the patient is not very comfortable during the examination, the probe is hand-held and there no built-in way to check fixation and eye alignment), the accuracy of measurements with the UBM may be poor, as it is confirmed by the lack of overlapping of corneal borders, while the edges of the ICPIOL are aligned, in the images provided where three scans are pasted together. Perfectly superimposable images are needed to achieve that grade of precision.

In all the eyes, the ACD (two UBM scans were taken to assess the reliability of the method), PACD, IL, S/IOEI, HA were measured. All measurements were made using the methods reported previously. ${ }^{11,12}$ All eyes were scanned by a single operator.

\section{Statistical analysis}

Data were collected on standardized case-report forms and then entered into a central database for analysis. There were no missing data in the analysis. Measures were compared using Pearson correlation and paired samples $t$ test. The level of significance was $P<0.05$.

\section{Results}

The mean power of the implanted ICPIOLs was $-15.11 \pm 4.45 \mathrm{D}$ (range -8.00 to $-20.50 \mathrm{D}$ ). No intraoperative complications were observed, except the slight iris pigment erosion near haptics due to surgical manipulation (Figure 1). There were no signs of postoperative complications, except incision leakage in one eye, which was resutured successfully 2-day postoperatively. The mean refractive spherical equivalent (SE) was $-16.04 \pm 5.46 \mathrm{D}$ (range -7.88 to $-22.88 \mathrm{D}$ ) preoperatively and $-1.11 \pm 0.54 \mathrm{D}(-0.38$ to $-2.25 \mathrm{D})$ postoperatively. The paired samples $t$ analysis showed a significance of SE reduction $(t=-8.80, P<0.01)$.

Table 1 shows the reliability and accuracy in measuring ACD using SP and UBM. The preoperative and postoperative anterior segment measurement data by the two methods are shown in Table 2. The ACD

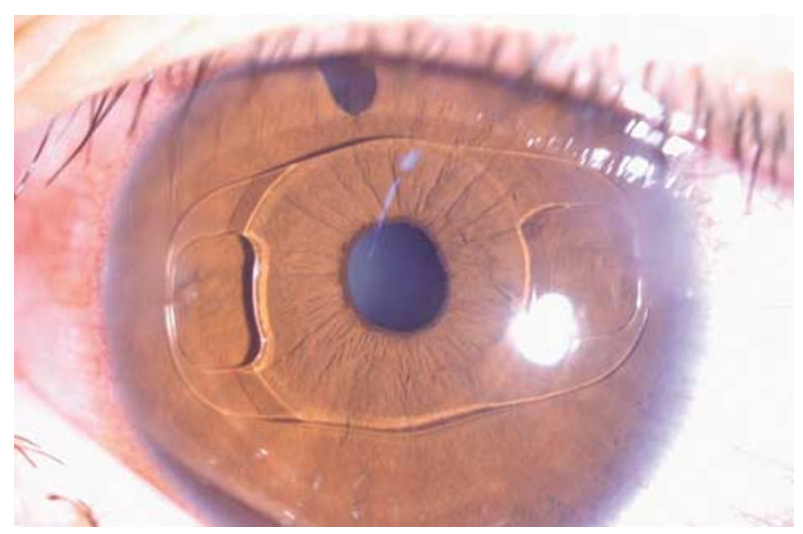

Figure 1 Slit lamp image of left eye in a patient implanted with VRSM50 model. The ICPIOL was clear and fixed horizontally. Slight iris pigment erosion near nasal haptic due to surgical manipulation can be seen.

Table 1 Reliability of SP and UBM in measuring ACD

\begin{tabular}{lccrrr}
\hline Parameter & $\mathrm{r}$ & P-value & Difference $(\mathrm{mm})$ & $\mathrm{t}$-value & P-value \\
\hline $\mathrm{SP}$ & 0.974 & 0.000 & $0.011 \pm 0.032$ & 1.048 & 0.325 \\
$\mathrm{UBM}$ & 0.986 & 0.000 & $-0.001 \pm 0.021$ & -0.207 & 0.841 \\
\hline
\end{tabular}

Abbreviations: ACD, the distance between the corneal endothelium and the anterior surface of the lens capsule preoperatively; SP, Scheimpflug photography; UBM, ultrasound biomicroscopy.

assessment, except the IL, proved well-correlated and there was no significant difference between the two methods, whereas the anterior segment parameters of PACD, IL, OEI, and HA measured by SP and UBM were uncorrelated, and significantly different. The mean difference between SOEI and IOEI using SP was $0.16 \mathrm{~mm}$, which was statistically significant $(t=2.68, P=0.03)$, and that using UBM was insignificant $(t=1.77, P=0.12)$.

$S P$ revealed that the inferior optic edge touched iris in two eyes implanted with VRSM50 model (Figure 2), and both the superior and inferior optic edge touched iris in four eyes implanted with VRSM60 model (Figure 3). Same findings of optic-iris touch were not observed by UBM (Figure 4). The two arms of the haptics near the site of iris entrapment showed indentation of the arms (Figures 5 and 6). However, no iris curvature distortion or pigment dispersion was observed.

\section{Discussion}

The significant SE reduction between the preoperative and postoperative periods in this study corroborates the efficacy of ICPIOL for high myopia. However, phakic eyes are more crowded than aphakic eyes. Therefore, it is essential to avoid touching sensitive intraocular tissues. Contact with the inner surface of the cornea can lead to endothelial cell loss. Contact with the iris can cause 
Table 2 Anterior segment measurements by SP and UBM in nine eyes

\begin{tabular}{lllllrr}
\hline Measurement & $A C D(\mathrm{~mm})$ & $P A C D(\mathrm{~mm})$ & $I L(\mathrm{~mm})$ & SOEI $(\mathrm{mm})$ & IOEI $(\mathrm{mm})$ & $H A(\mathrm{~mm})$ \\
\hline SP & $3.10 \pm 0.14$ & $1.88 \pm 0.09$ & $0.76 \pm 0.13$ & $0.23 \pm 0.23$ & $0.07 \pm 0.13$ & $0.90 \pm 0.17$ \\
UBM & $3.07 \pm 0.11$ & $1.99 \pm 0.12$ & $0.67 \pm 0.06$ & $0.58 \pm 0.24$ & $0.41 \pm 0.22$ & $1.45 \pm 0.13$ \\
$R$ & 0.94 & 0.57 & 0.18 & 0.56 & 0.03 & 0.42 \\
$P$-value & 0.00 & 0.11 & 0.64 & 0.12 & 0.95 & 0.26 \\
Mean difference & 0.03 & -0.11 & 0.09 & -0.36 & -0.33 & -0.55 \\
$t$-value & 1.95 & -3.40 & 2.09 & -4.82 & -4.02 & -9.97 \\
$P$-value & 0.09 & 0.01 & 0.07 & 0.00 & 0.00 & 0.00 \\
\hline
\end{tabular}

Abbreviations: ACD, the distance between the corneal endothelium and the anterior surface of the lens capsule preoperatively; HA, the shortest distance between the farthest point of the ICPIOL haptics and the angle of anterior chamber; ICPIOL, iris-claw phakic intraocular lens; IL, the distance between the posterior surface of the ICPIOL and the anterior surface of the lens capsule; IOEI, the distance between the inferior optic edge and the iris; PACD, the distance between the corneal endothelium and the anterior surface of the ICPIOL; SOEI, the distance between the superior optic edge and the iris; SP, Scheimpflug photography; UBM, ultrasound biomicroscopy.

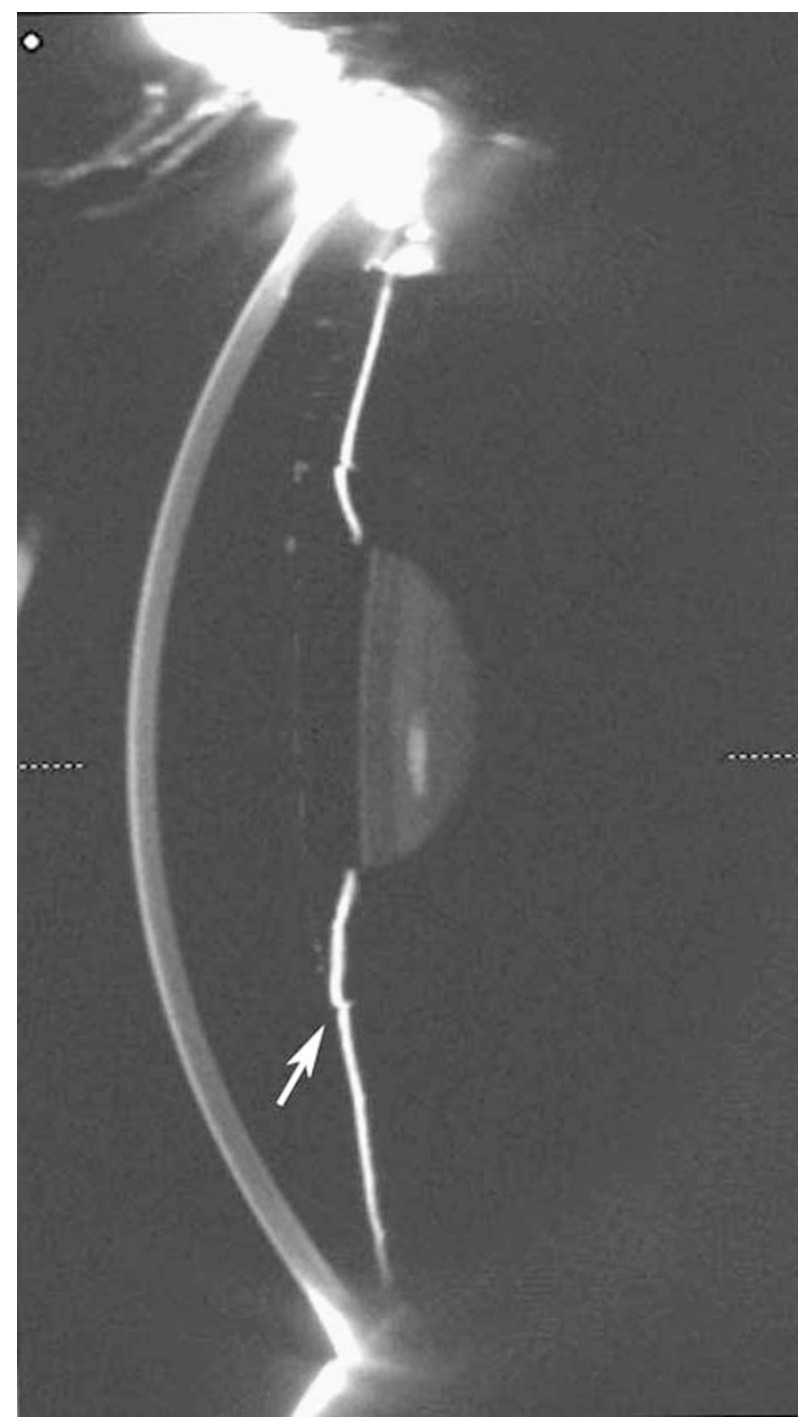

Figure 2 Scheimpflug image of left eye in a patient. The distance between the SOEI was $0.30 \mathrm{~mm}$, the inferior optic edge touched iris (arrow).

pigment dispersion and a chronic inflammatory reaction, and contact with the crystalline lens may result in cataract. We think precise evaluation of the ICPIOL's

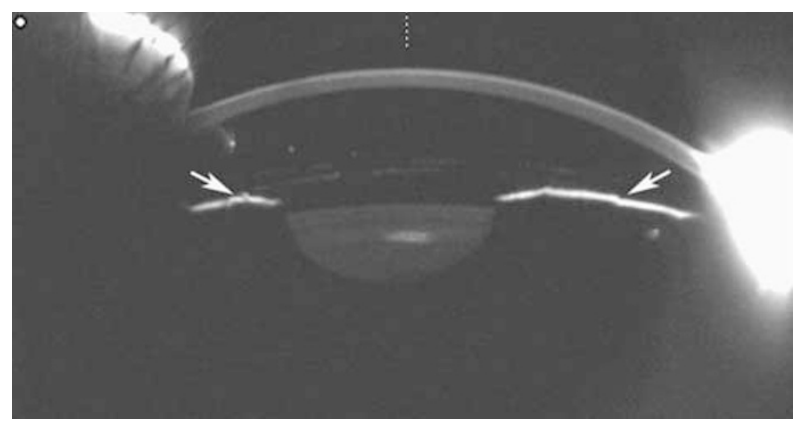

Figure 3 Scheimpflug image of right eye in a patient implanted with VRSM60 model. Both the superior and inferior optic edge touched iris (arrow).

relationship to ocular structures in different body positions is important for the early diagnosis of eventual alterations and complications. The advantages of the SP technique are the fast noncontact-acquisition of data and the high image quality, with great depth of focus in upright position. UBM can be performed in a noninvasive way, but there is contact. The advantages of the UBM technique are the high-definition images of anterior segment, including the posterior surface of iris and angle of anterior chamber in supine position. Thus, analysis by SP and UBM may be useful in the management of patients with ICPIOLs. To our knowledge, this is the first study to date to investigate in situ the intraocular position of the Verisyse ICPIOL using SP and UBM.

The accuracy of a measuring instrument is an essential factor when selecting a device for clinical purposes. This study shows the high precision and good reliability of both SP and UBM. The physical conditions in which the measurements were taken might affect the results in both instruments. To obtain the result that can reflect the real position of ICPIOL in clinical practice, cycloplegia was not used in this study. Without cycloplegia, measurements may be influenced by changes in the accommodative state. However, comparisons between Orbscan and IOLMaster without cycloplegia have been 


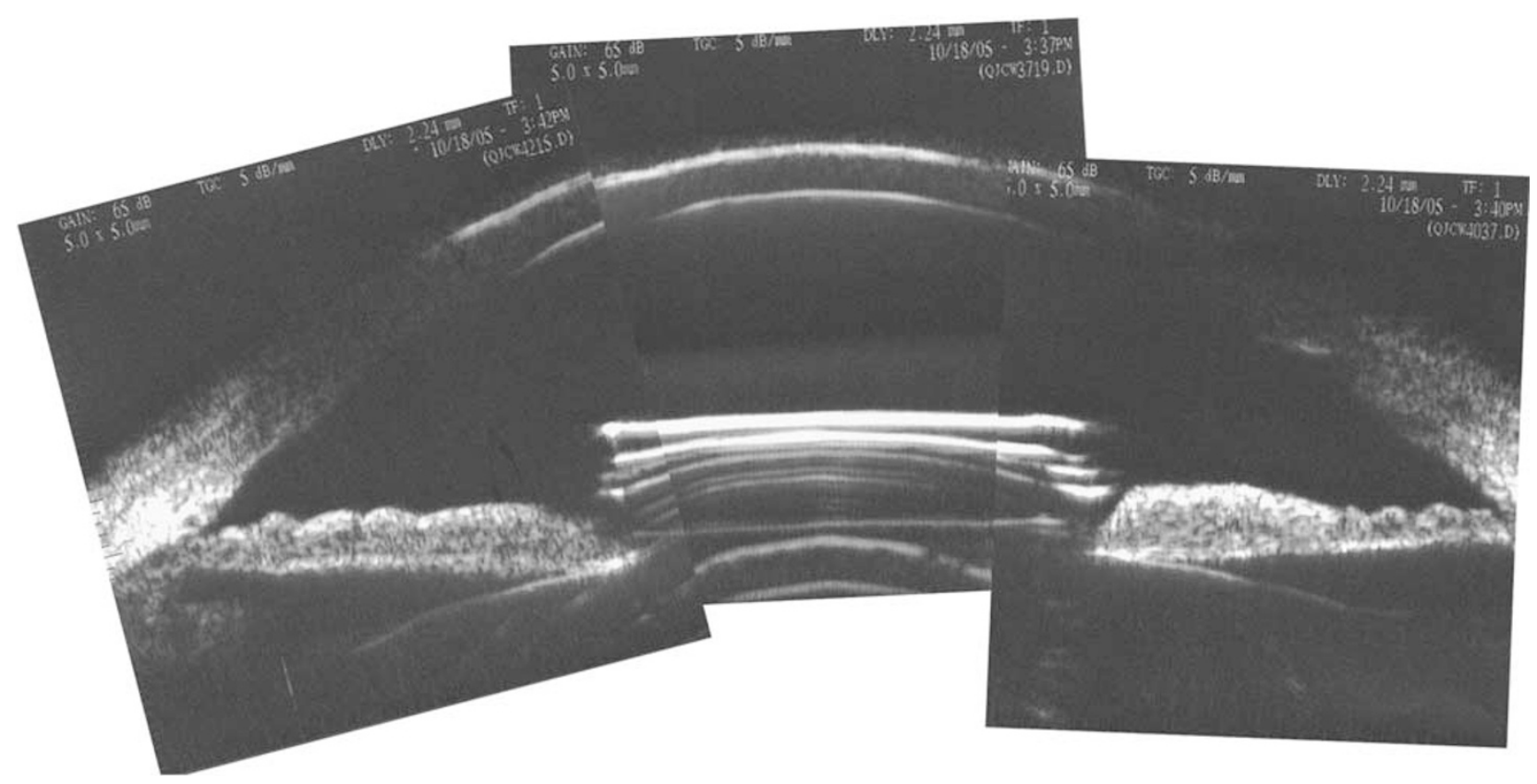

Figure 4 Postoperative composite picture of 3 vertical UBM echogram of the ICPIOL of left eye in a patient. The ICPIOL did not touch iris or crystalline lens.

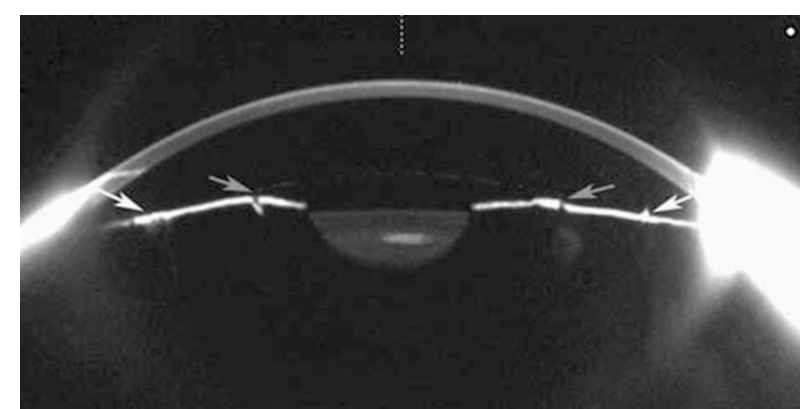

Figure 5 Scheimpflug image of left eye in a patient. The lateral optic edge did not touch iris (grey arrow). The 2 arms of the haptics near the site of iris entrapment showed indentation of the arms (white arrow). Adequate space was maintained between the haptics and the corneal endothelium, angle.

reported to produce comparable ACD measurements. ${ }^{13,14}$ In addition, various factors, including the experience of the operator and the user technique might affect the measurements in both techniques. Nevertheless, the ACD assessment using the two methods proved well correlated. On a clinical point of view, the difference $(0.03 \mathrm{~mm})$ seems narrow enough to make it unimportant to a certain extent, unless high accuracy is demanded for formulas derived from measurements by a particular biometric method. In addition, one may ask whether the parameter measured in an optical system using light compares favorably with that measured in a system using ultrasound. If there existed significant differences between SP and UBM measures in the determination of distances between ICPIOL and ocular structures, appropriate correction factors are needed. In this study, the between-method difference in the measurement of ACD was statistically insignificant, which shows the high precision of both instruments. Therefore, we consider that measurements performed using SP and UBM for these patients will yield equivalent and reliable results in evaluating the distance from the ICPIOL to the endothelium, iris, and lens in the central region.

The assessment of the PACD, IL, OEI, and HA using the two methods proved uncorrelated, and significantly different, except for the IL. SP showed that the inferior optic edge touched iris, but the iris curvature was undisturbed (Figure 2), which presented no existence of indentation within the touched area of iris, whereas no optic-iris touch was observed by UBM (Figure 4). The explanations for the between-method difference might be the following: (1) pronounced natural iris arch preoperatively. As the preoperative examination indicated, these eyes had a normal natural iris arch, this probability can be ruled out. (2) pupillary block.

However, this explanation would be refuted because the postoperative IOP remained normal. (3) confusion of artifacts. UBM was reported to promote artifacts at the posterior face of ICPIOL, which presented difficulty in finding the exact point from the posterior ICPIOL face in evaluating IL and OEI. Fortunately, formation of artifacts did not reach a certain extent to confuse the posterior ICPIOL face in this study. (4) change in body position explaining the differences between optic-iris touch 


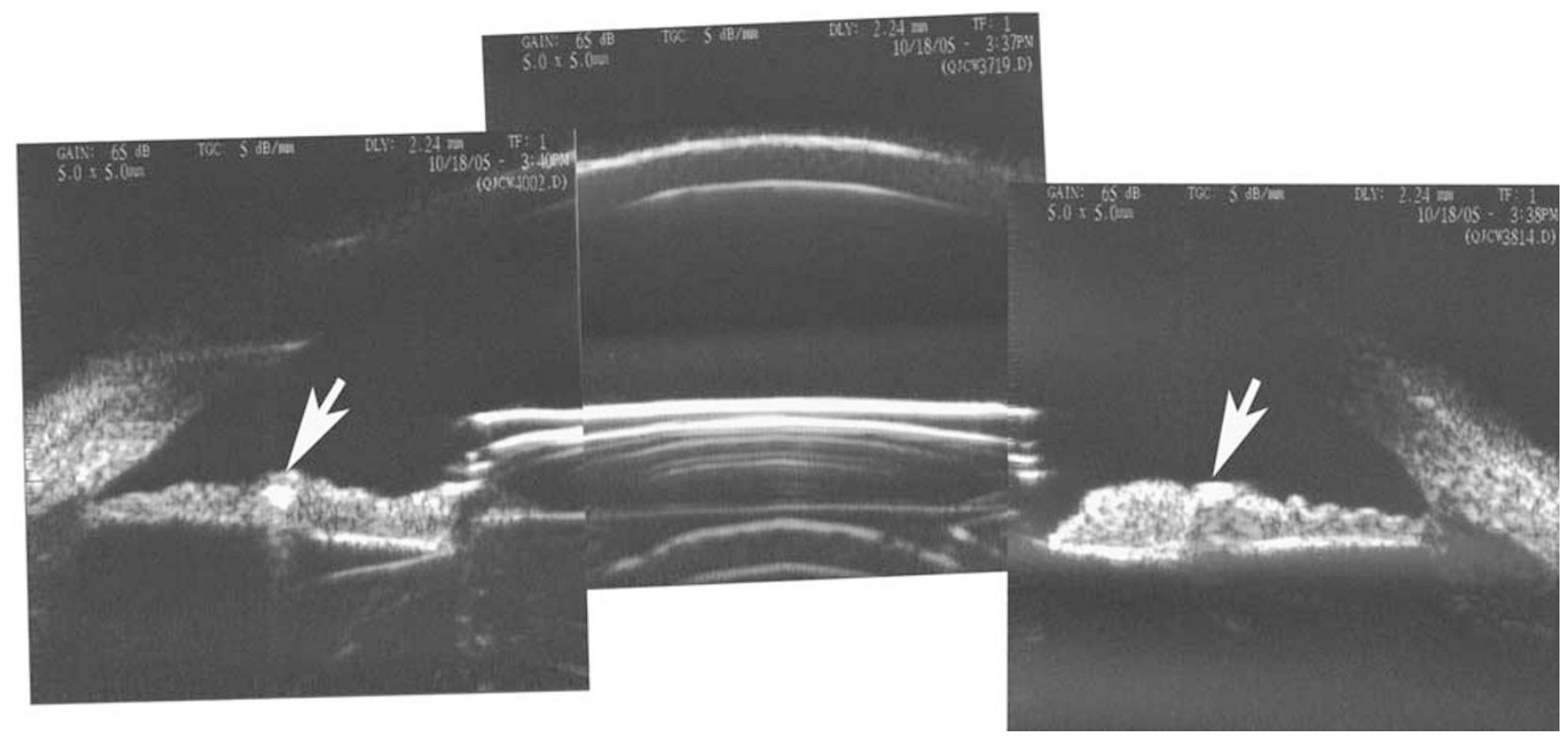

Figure 6 Postoperative composite picture of 3 horizontal UBM echograms of the ICPIOL of left eye in a patient showing the ICPIOL indentation into the iris (arrow) and the shadowing behind it through the iris entrapment. The ICPIOL did not touch corneal endothelium, angle, or crystalline lens.

registered with two instrumentations. Contrary to supine position while UBM was performed, patients were asked to keep upright head position during SP examination, and the ICPIOL moved downward, or rotated along its lateral axis under the influence of gravity. It is similar to the condition that a photo frame will tilt when it is suspended on wall. The IOEI was less than SOEI in upright position (Figure 2), whereas they were not significantly different in supine position. The position of ICPIOL inside the eyes simultaneously depends on the two haptics and the inferior optic edge because of gravitational effects in upright position. (5) secondary to inadequate vaulting of the VRSM60 model. Both the superior and inferior optic edge touched iris in two patients implanted with VRSM60 (Figure 3). The vault was relatively inadequate due to the optical diameter of $6.0 \mathrm{~mm}$. On account of the explanation (4) and (5), a peripheral iridectomy is suggested to be performed as a precaution though there is space between the lateral optic edge and iris. In addition, surgeons should be aware that individuals with a natural highly vaulted iris might be particularly susceptible to ICPIOL haptics indentation and optic-iris touch. Further study is required to evaluate the potential complications and the relation of the optic edge with the iris. In future, refinements in ICPIOL design, that is enlarging footplate, shortening the haptics or increasing the ICPIOL vaulting, might eliminate these potentials in Chinese eyes.

Despite the ICPIOL's position variations with change of body position, SP and UBM revealed that adequate space was maintained between the ICPIOL and the corneal endothelium, angle, and crystalline lens.

However, these measurements of PACD and IL are not similar to those reported in Caucasian myopic eyes. Baumeister $e t a l^{15}$ reported that the PACD and IL using SP were $2.48 \pm 0.25$ and $0.46 \pm 0.15 \mathrm{~mm}$, respectively. Pop et al $^{16}$ reported that the PACD and IL using UBM ranged from 2.11 to 2.44 and 0.78 to $0.93 \mathrm{~mm}$, respectively. The explanations for the difference include the following: (1) racial difference in ocular anatomy. For instance, Caucasian eyes had relatively great ACD preoperatively. (2) examination condition not same. Baumeister had patients examined under cycloplegia according to various research purposes. Because of the adequate space maintained, it thus probably eliminates the potential for complications secondary to the ICPIOL-intraocular tissue interaction.

As we expected, the HA with SP was $0.55 \mathrm{~mm}$ shorter compared with UBM. This is due to the major disadvantage of SP that it is a purely optical technique requiring clear optical media. Measurement of HA as performed here encounters the difficulty that SP does not allow an observation of the non-transparent limbus when trying to determine precisely the border of the angle of anterior chamber, and there is a need to create the point at which the angle would presumably be. Chinese have been reported frequently to have smaller eyes compared with other races. ${ }^{17,18}$ It is likely that in Chinese eyes, the enclavation points probably are situated more peripheral than in Caucasian eyes. This could lead to lens movement and accompanying pupil movement, resulting in corneal endothelial damage or cataract 
formation. In this study, the HA with UBM was $1.47 \mathrm{~mm}$. It is unlikely that the corneal endothelium and the angle of anterior chamber will be damaged during pupil reaction because the midperipheral iris tissue enclavated by the haptics is relatively stable.

The fixation method of ICPIOL involves enclavation of two diametrically opposite haptics on midperipheral iris stroma. The question rooted from the ICPIOL is whether the enclavation causes iris tissue damage, especially in Chinese eyes in which smaller corneal diameter and darker iris pigmentation are usually observed. ${ }^{17,18}$ The SP and UBM showed that the haptics appeared to indent iris tissue, and the magnitude of the indentation increased closer to the site of iris entrapment (Figures 1, 5, and 6), and that may present an area from which pigment dispersion could occur, and prone to cause iritis. However, the iris curvature appeared to be undisturbed except slight fold on itself within a small area of the iris entrapment, neither pigment dispersion nor iritis was noted. Complications from pigment dispersion, ie pigmentary dispersion glaucoma, have not occurred in this small group of eyes with limited follow-up. This is consistent with those observed in Caucasians. ${ }^{16}$ The ICPIOL does not affect the pigment dispersion in the anterior chamber as long as it is appropriately fixed because the amount of melanin granules in the anterior stroma of the iris is less than in the iris pigment epithelium. However, the changes of pupil reaction remain to be studied due to the limitation of both instruments.

This study adds to our previous knowledge of ICPIOL measurement differences with various devices available. In analysis of the management of patients with an ICPIOL, the two methods are not equivalent. The different body positions are the main reason for the statistically significant difference between the methods used in measurements of ICPIOL's position. In conclusion, analysis of measurements by SP and UBM reveals the ICPIOL's position variations with changes of body position. Nevertheless, adequate space was maintained between the ICPIOL and the corneal endothelium, angle, and crystalline lens. The ICPIOL implanted in phakic eyes seems a safe alternative for the correction of high myopia. Further investigations of larger number of subjects and longer follow-up periods are warranted.

\section{References}

1 Landesz M, Worst JGF, van Rij G. Long-term results of correction of high myopia with an iris claw phakic intraocular lens. J Refract Surg 2000; 16: 310-316.
2 Budo C, Hessloehl JC, Izak M, Luyten GP, Menezo JL, Sener BA et al. Multicenter study of the Artisan phakic intraocular lens. J Cataract Refract Surg 2000; 26: 1163-1171.

3 Maloney RK, Nguyen LH, John ME. Artisan phakic intraocular lens for myopia; short-term results of a prospective, multicenter study; the Artisan Lens Study Group. Ophthalmology 2002; 109: 1631-1641.

4 Dick HB, Alio J, Bianchetti M, Budo C, Christiaans BJ, El-Danasoury MA et al. Toric phakic intraocular lens; European multicenter study. Ophthalmology 2003; 110: 150-162.

5 Saxena R, Landesz M, Noordzij B, Luyten GP. Three-year follow-up of the Artisan phakic intraocular lens for hypermetropia. Ophthalmology 2003; 110: 1391-1395.

6 Tehrani M, Dick HB, Schwenn O, Blom E, Schmidt AH, Koch HR. Postoperative astigmatism and rotational stability after Artisan toric phakic intraocular lens implantation. J Cataract Refract Surg 2003; 29: 1761-1766.

7 Menezo JL, Cisneros AL, Rodriguez-Salvador V. Endothelial study of iris-claw phakic lens: four year follow-up. J Cataract Refract Surg 1998; 24: 1039-1049.

8 Menezo JL, Martinez MC, Cisneros AL. Iris-fixated Worst claw versus sulcus-fixated posterior chamber lenses in the absence of capsular support. J Cataract Refract Surg 1996; 22: 1476-1484.

9 Menezo JL, Avino JA, Cisneros A, Rodriguez-Salvador V, Martinez-Costa R. Iris claw phakic intraocular lens for high myopia. J Refract Surg 1997; 13: 545-555.

10 Coppens JE, van den Berg TJTP, Budo CJ. Biometry of phakic intraocular lens using Scheimpflug photography. J Cataract Refract Surg 2005; 31: 1904-1914.

11 Pavlin CJ, Harasiewicz K, Foster FS. Ultrasound biomicroscopy of anterior segment structures in normal and glaucoma eyes. Am J Ophthalmol 1992; 113: 381-389.

12 Wang N, Lai M, Chen X, Zhou W. Quantitative real time measurement of iris configuration in living human eyes. Chin J Ophthalmol 1998; 34(5): 369-372.

13 Rabsilber TM, Becker KA, Frisch IB, Auffarth GU. Anterior chamber depth in relation to refractive status measured with the Orbscan II Topography System. J Cataract Refract Surg 2003; 29: 2115-2121.

14 Reddy AR, Pande MV, Finn P, El-Gogary H. Comparative estimation of anterior chamber depth by ultrasonography, Orbscan II, and IOLMaster. J Cataract Refract Surg 2004; 30: 1268-1271.

15 Baumeister M, Buhren J, Kohnen T. Position of anglesupported, iris-fixated, and ciliary sulcus-implanted myopic phakic intraocular lenses evaluated by Scheimpflug photography. Am J Ophthalmol 2004; 138: 723-731.

16 Pop M, Mansour M, Payette Y. Ultrasound biomicroscopy of the iris-claw phakic intraocular lens for high myopia. J Refract Surg 1999; 15: 632-635.

17 Matsuda LM, Woldorff CL, Kame RT, Hayashida JK Clinical comparison of corneal diameter and curvature in Asian eyes with those of Caucasian eyes. Optom Vis Sci 1992; 69: 51-54.

18 Jeong S, Lemke BN, Dortzbach RK, Park YG, Kang HK. The Asian upper eyelid; an anatomical study with comparison to the Caucasian eyelid. Arch Ophthalmol 1999; 117: $907-912$. 